\title{
Protoplast Lysis and Inhibition of Growth of Bacillus licheniformis by Bacitracin
}

\author{
JOHN E. SNOKE AND NEAL CORNELL ${ }^{1}$ \\ Department of Biological Chemistry, School of Medicine, University of California, \\ Los Angeles, California
}

Received for publication 24 September 1964

\begin{abstract}
Snoke, John E. (University of California, Los Angeles), and Neal Cornell. Protoplast lysis and inhibition of growth of Bacillus licheniformis by bacitracin. J. Bacteriol. 89:415-240. 1965.-The growth of Bacillus licheniformis is inhibited by bacitracin, the antibiotic which the organism itself produces. The effect of the antibiotic is confined to the early growth phase of the organism. Bacitracin will produce a rapid lysis of protoplasts of $B$. licheniformis and Micrococcus lysodeikticus. The lytic process requires cadmium or zinc ions, and the rate and extent of lysis depends upon the amount of bacitracin and metal ion added. Although $B$. licheniformis and $M$. lysodeikticus differ markedly in their sensitivity to bacitracin, the protoplasts from these two organisms are equally susceptible to lysis by the antibiotic.
\end{abstract}

The antibiotic bacitracin has been shown to possess a variety of biological activities. In addition to its antibiotic activity against susceptible organisms, bacitracin induces cell lysis (Crawford and Abraham, 1957; Smith and Weinberg, 1962), protoplast formation (Abraham, 1957), accumulation of cell-wall intermediates (Abraham and Newton, 1958; Park, 1958, 1960), and it prevents the incorporation of labeled amino acids into cell-wall mucopeptides (Park, 1958; Mandelstam and Rogers, 1959). These findings suggest that the antibiotic interferes with the synthesis of cell wall. In addition, it has been shown that the synthess of induced enzymes is also inhibited by bacitracin (Creaser, 1955; Gale and Folkes, 1955; Smith and Weinberg, 1962).

We have observed that under certain conditions the growth of Bacillus licheniformis can be inhibited by bacitracin, the antibiotic which the organism itself produces. The levels of antibiotic required to inhibit the growth of $B$. licheniformis are considerably higher than those required for sensitive organisms. It was considered possible that the low sensitivity of $B$. licheniformis was due to the impermeability of the cell to the antibiotic. Since $B$. licheniformis is readily transformed into protoplasts (Snoke, 1961), it appeared possible to test this hypothesis by studying the effect of the antibiotic on protoplasts. When bacitracin was added to suspensions of protoplasts, a rapid lysis of the bacterial structure was observed.

${ }^{1}$ Present address: Department of Biological Chemistry, Harvard University Medical School, Boston, Mass.
The two properties of bacitracin, the antibiotic activity toward $B$. licheniformis and the lytic activity on protoplasts, are described in this report.

\section{Materials and Methods}

The density of bacterial or protoplast suspensions was determined by measuring the optical density at $620 \mathrm{~m} \mu$ with cylindrical cells $15 \mathrm{~mm}$ in diameter. When necessary, samples were diluted so that the optical-density readings were in a proportional range. The two organisms used were the bacitracin-producing strain of $B$. licheniformis (ATCC 10716) and a strain of Micrococcus lysodeikticus kindly supplied by John M. Pickett. Spores of $B$. licheniformis were obtained in the manner outlined by Bernlohr and Novelli (1960). Initiation of cultures was made by transferring approximately $10^{9}$ spores to $5 \mathrm{ml}$ of $0.5 \%$ Peptone, $0.4 \%$ Yeast Extract (Difco), and 0.3\% Beef Extract (Difco). After heating at 60 to $70 \mathrm{C}$ for $20 \mathrm{~min}$, the tube was shaken at $36 \mathrm{C}$ for $18 \mathrm{hr}$. This culture was adjusted to an optical density of $2.0\left(9 \times 10^{8}\right.$ cells per $\left.\mathrm{ml}\right)$ and then was used to make a $0.5 \%$ inoculum in a synthetic medium. Samples $(10 \mathrm{ml})$ of the inoculated cultures in 50 -ml Erlenmeyer flasks were agitated at $36 \mathrm{C}$ by a reciprocating shaker at 120 cycles per min. In some cases, cultures were grown in 1-liter flasks containing $150 \mathrm{ml}$ of medium.

The synthetic medium, which was a modification of that described by Hendlin (1949), contained in 1 liter; $12.0 \mathrm{~g}$ of L-glutamic acid, $2.0 \mathrm{~g}$ of citric acid, $0.1 \mathrm{~g}$ of $\mathrm{MgSO}_{4}, 0.5 \mathrm{~g}$ of $\mathrm{K}_{2} \mathrm{HPO}_{4}$, and 0.025 g of $\mathrm{FeCl}_{2}$, adjusted to $p \mathrm{H} 7.0$ with $\mathrm{NaOH}$.

$M$. lysodeikticus was maintained on Nutrient Agar (Difco) from which transfers were made in 5 $\mathrm{ml}$ of $0.5 \%$ Peptone, $0.4 \%$ Yeast Extract, and 
$0.3 \%$ Beef Extract. After shaking for $8 \mathrm{hr}$ at $36 \mathrm{C}$, $0.75 \mathrm{ml}$ was transferred into $150 \mathrm{ml}$ of the same medium which was then shaken at $36 \mathrm{C}$ for $16 \mathrm{hr}$.

Cells from $16-\mathrm{hr} B$. licheniformis cultures were harvested, washed twice with $0.85 \% \mathrm{NaCl}$, and suspended in a volume of water to give an optical

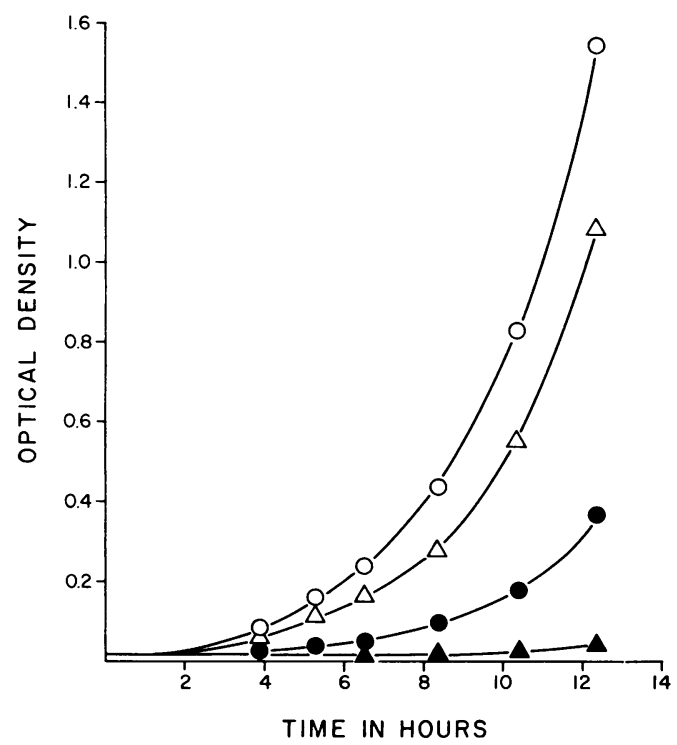

Fig. 1. Effect of bacitracin on the growth of Bacillus licheniformis. Experimental conditions were as described in Materials and Methods, except that bacitracin was added to the incubation flasks. The amount of bacitracin present in each flask was: none $(\bigcirc), 0.5$ unit per $\mathrm{ml}(\triangle), 1.0$ unit per $\mathrm{ml}(\mathbf{\odot})$, and 5.0 unit per $\mathrm{ml}(\mathbf{\Delta})$. Cadmium chloride was present at $10^{-4} \mathrm{M}$.

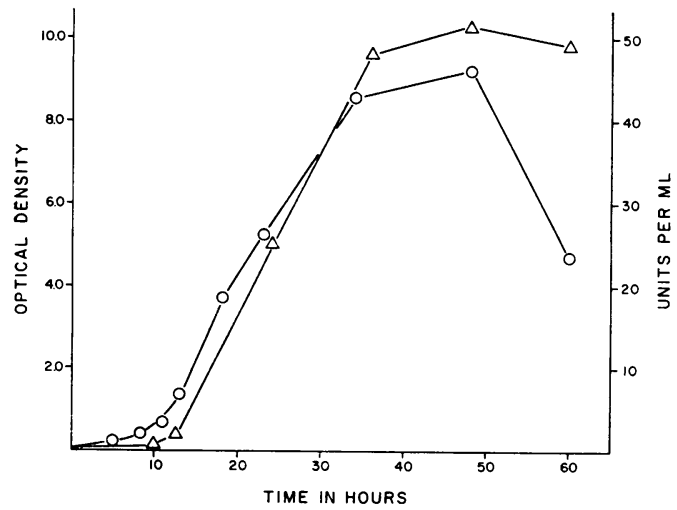

Fig. 2. Growth and bacitracin production in synthetic medium. Experimental conditions were as described in Materials and Methods, except that the culture flask was a 1-liter Florence flask containing $200 \mathrm{ml}$ of medium, and the cells were grown at $32 \mathrm{C}$. The optical density of the medium is indicated by $\mathrm{O}$; the antibiotic concentration by $\triangle$. density of 50. Protoplasts were prepared by adding the following to $5 \mathrm{ml}$ of cell suspension: $0.36 \mathrm{ml}$ of $0.1 \mathrm{~m}$ potassium phosphate buffer, $p \mathrm{H} 7.0 ; 1.83$ $\mathrm{ml}$ of $2.0 \mathrm{M}$ sucrose; and $0.09 \mathrm{ml}$ of a lysozyme solution $(20 \mathrm{mg} / \mathrm{ml})$. The suspension was incubated at room temperature for $1 \mathrm{hr}$, at which time all of the cells were converted to protoplasts as judged by phase-contrast microscopy. The protoplasts were collected by centrifugation, washed once with $0.5 \mathrm{M}$ sucrose, resuspended in $5 \mathrm{ml}$ of $0.5 \mathrm{~m}$ sucrose, and then treated with $10 \mu \mathrm{g}$ of crystalline deoxyribonuclease (Worthington Biochemical Corp., Freehold, N.J.). Protoplasts were prepared from $M$. lysodeikticus in the same manner except that the harvested cells were suspended in water to give an optical density of 100 , and the protoplast preparation, washing, and suspension were carried out in $1.0 \mathrm{~m}$ sucrose. The determination of bacitracin was carried out by the turbidimetric method of Darker et al. (1948) with Staphylcoccus aureus ATCC 10537 as the test organism. Bacitracin used in this study was obtained from Nutritional Biochemicals Corp., Cleveland, Ohio, and contained 70 units per $\mathrm{mg}$.

\section{Results}

Bacitracin, an antibiotic produced by $B$. licheniformis, inhibited the growth of the same organism (Fig. 1). To be certain that the observed inhibition was actually a case of an antibiotic being effective against the same organism which produces it, bacitracin was purified from the same strain of $B$. licheniformis that was used in the growth studies. When bacitracin isolated from cultures of ATCC 10716 was tested, results identical to those in Fig. 1 were obtained.

The concentration of bacitracin required for the inhibition of growth was approximately 50 times larger than that required for organisms such as $S$. aureus, so that $B$. licheniformis could not be considered a particularly sensitive or ganism. However, in cultures bacitracin accumulated in amounts of 50 units per $\mathrm{ml}$ and higher; thus, the results raised the question as to whether the growth of the organism in culture is limited by its production of the antibiotic.

Figure 2 shows an example of the growth of $B$. licheniformis and the corresponding production of bacitracin. As the antibiotic accumulates in the medium, the growth of the organism declines, reaching a stationary phase at approximately the same time that the maximal level of antibiotic is reached. These results suggested a causal role of the antibiotic in the cessation of bacterial growth. When a test was made of the hypothesis that the production of bacitracin was responsible for the cessation of bacterial growth, it was found that the correspondence between the decline of growth and the accumulation of antibiotic was fortuitous. If the addition of bacitracin to a cul- 
ture of $B$. licheniformis was delayed several hours after the culture was started, the antibiotic had no effect on the subsequent growth. If the antibiotic is to be effective in suppressing growth, it must be present at the initiation of the culture or shortly thereafter (Fig. 3). If the addition of bacitracin was made $14 \mathrm{hr}$ after the initiation of the culture, the growth of the culture was indistinguishable from the culture containing no bacitracin.

Although bacitracin is active against $B$. licheniformis under certain conditions, the organism must be considered to be relatively resistant. As it appeared possible that the basis for the resistance might be due to the slow permeation of the antibiotic into the cell, experiments testing the effect of bacitracin on protoplasts were considered. It was observed that the addition of bacitracin and certain metal ions caused a rapid lysis of protoplasts of $B$. licheniformis suspended in $0.5 \mathrm{~m}$ sucrose. Neither bacitracin nor the metal ions alone had any lytic action on the protoplast. Lysis was observed by measuring the decrease in optical density at four different concentrations of the antibiotic (Fig. 4). The initial rate of protoplast lysis was approximately proportional to the concentration of bacitracin present, and the extent of lysis also depended upon the amount of bacitracin added (some variability in the extent of lysis was also observed with different preparations of protoplasts).

Cadmium and zinc ions, which are required for the microbiological activity of bacitracin (Weinberg, 1959; Adler and Snoke, 1962), were active in promoting the protoplast lytic activity of bacitracin. Magnesium, calcium, and manganese, as well as various monovalent cations, were without effect. The effect of cadmium ion concentration on the lysis of protoplasts is shown in Fig. 5. As was the case with different bacitracin concentrations, the extent of protoplast lysis also depended upon the amount of cadmium ions present. The partial lysis observed in the presence of small amounts of cadmium suggested that the cadmium became bound during the disruption of the protoplast, and that lysis stopped due to the lack of metal ion. However, if additional cadmium was added to the system containing partially lysed protoplasts, the additional lysis was only slight. The additions of cadmium are indicated by the arrows in Fig. 5. In these cases, the extent of lysis was still less than that of the other experiments; thus, it would appear that changes in the system in addition to the binding of cadmium were involved.

The finding that the extent of lysis of protoplasts depends upon the amount of bacitracin added suggested that the bacitracin became inactive as the result of the lytic process. Likewise, it

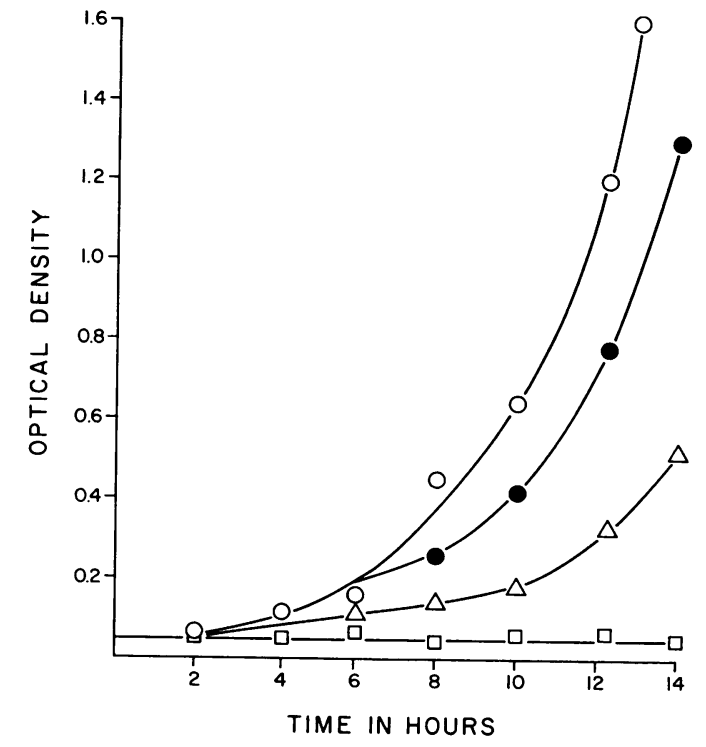

FIG. 3. Effect of bacitracin added to growing cul tures of Bacillus licheniformis. Experimental conditions were as described in Materials and Methods, except that 500 units of bacitracin were added to each flask containing $10 \mathrm{ml}$ of medium. The abscissa refers to the time after the culture was initiated. The time at which the bacitracin was added was: zero $h r(\square), 2 h r(\triangle)$, and $6 \mathrm{hr}(\mathbf{\bigcirc})$. Cadmium chloride was present at $10^{-4} \mathrm{M}$. The control, $\bigcirc$, was the culture to which bacitracin was not added.

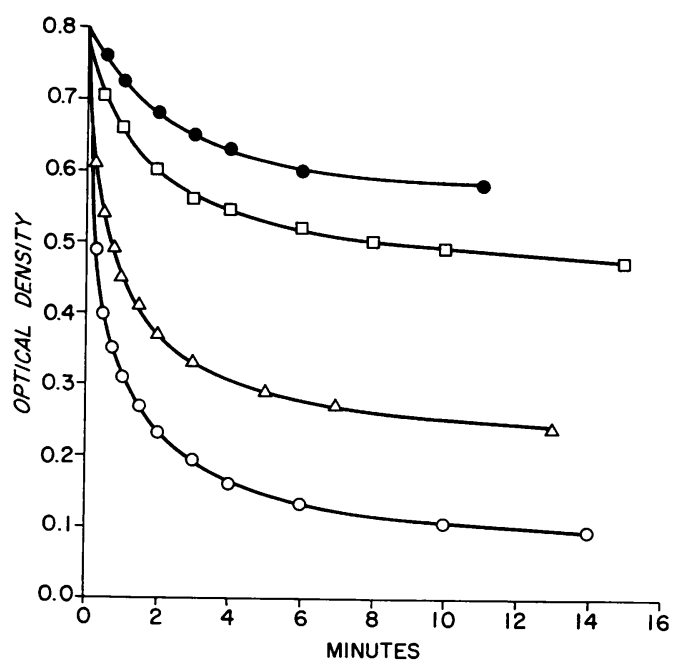

Fig. 4. Lysis of protoplasts of Bacillus licheniformis by bacitracin. The incubation mixture, 4.0 $m l$ ( $p H$ 8.5), contained $0.5 \mathrm{M}$ sucrose, $0.025 \mathrm{M}$ Tris buffer, $1.25 \times 10^{-4} \mathrm{M} \mathrm{CdCl}_{2}, 0.2 \mathrm{ml}$ of a protoplast suspension, and bacitracin. The lysis of protoplasts was initiated by the addition of bacitracin. The amount of antibiotic (units per milliliter) in each system was: $25.0(\mathrm{O}), 8.0(\triangle), 2.5(\square)$, and 1.25 (O). 


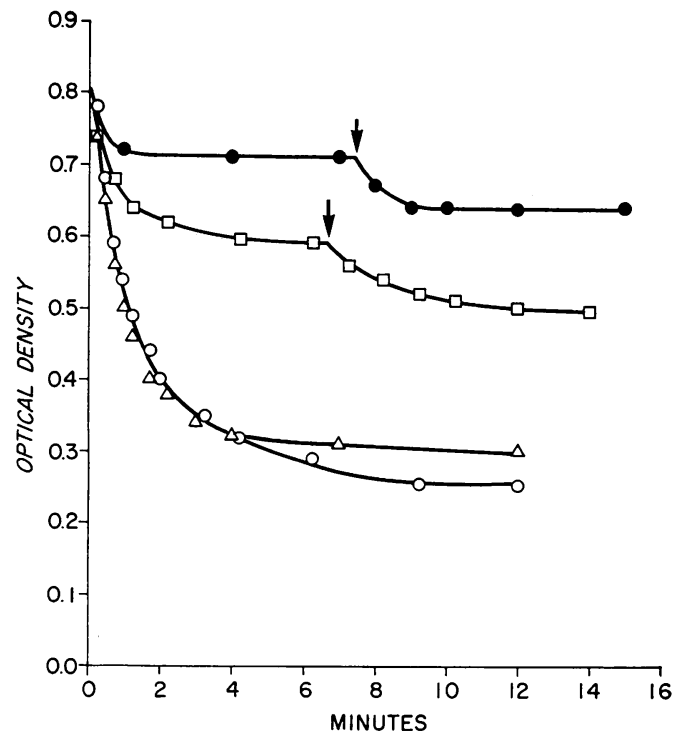

Fig. 5. Effect of cadmium concentration on the lysis of protoplasts by bacitracin. The incubation mixture, $4.0 \mathrm{ml}$ ( $\mathrm{pH} \mathrm{8.5)}$, contained $0.5 \mathrm{M}$ sucrose, 0.025 M Tris buffer, $0.2 \mathrm{ml}$ of a protoplast suspension of Bacillus licheniformis, 2.5 units of bacitracin per $\mathrm{ml}$, and various amounts of $\mathrm{CdCl}_{2}$. The amount of $\mathrm{CdCl}_{2}$ present initially in each system was: $4.0 \times$ $10^{-4} M(O), 1.25 \times 10^{-4} M(\triangle), 0.5 \times 10^{-4} M(\square)$, and $0.25 \times 10^{-4} M(0)$. At the time indicated by the arrows, $\mathrm{CdCl}_{2}$ was added to give a concentration of $1.25 \times 20^{-4} \mathrm{M}$.

TABLE 1. Recovery of bacitracin after lysis of protoplasts

\begin{tabular}{l|c|c|c|c}
\hline \multirow{2}{*}{ Expt } & $\begin{array}{c}\text { Bacitracin } \\
\text { added } \\
\text { units/ml) }\end{array}$ & $\begin{array}{c}\text { Cadmium } \\
\left(\mathbf{M} \times 10^{4}\right)\end{array}$ & $\begin{array}{c}\text { Protoplast } \\
\text { lysis }\end{array}$ & $\begin{array}{c}\text { Bacitracin } \\
\text { recovered } \\
\text { units/ml) }\end{array}$ \\
\cline { 2 - 5 } 1 & & & $\%$ & \\
\cline { 2 - 4 } 2 & 25.0 & 1.25 & 96 & 27.0 \\
3 & 8.0 & 1.25 & 78 & 9.6 \\
4 & 2.5 & 1.25 & 34 & 2.5 \\
$5^{*}$ & 1.25 & 1.25 & 29 & 1.6 \\
6 & 5.0 & 1.25 & - & 5.6 \\
7 & 5.0 & 1.25 & 75 & 4.4 \\
8 & 5.0 & 0.36 & 38 & 4.9 \\
9 & 5.0 & 0.18 & 23 & 4.7 \\
& 5.0 & 0.05 & 5 & 4.8 \\
\hline
\end{tabular}

* Protoplasts were lysed in water before the addition of bacitracin.

appeared possible that the limited lysis observed at low cadmium concentrations was also the result of bacitracin inactivation. An attempt to demonstrate this inactivation was carried out by measuring the antibiotic activity of bacitracin after the protoplast lysis had stopped. In experiments similar to those shown in Fig. 4 and 5, protoplasts were lysed with different amounts of bacitracin and cadmium. When the lytic process stopped, samples of the incubation mixture were diluted in water and assayed for their antibiotic activity. The results obtained (Table 1 ) demonstrated that in all cases the antibiotic was not destroyed. These experiments do not exclude the possibility that the lytic activity of bacitracin is lost as a result of the antibiotic being bound, but that this association is then reversed during the microbiological assay.

The initial rate of protoplast lysis was determined at different $p \mathrm{H}$ values. The marked inhibition observed with most of the buffer systems tested prevented an evaluation of the rate at $p \mathrm{H}$ values below 7 . The lysis was inhibited strongly by a variety of salts. The percentage decrease in the rate of lysis observed in the presence of $0.01 \mathrm{M}$ $\mathrm{NH}_{4} \mathrm{Cl}, \mathrm{KCl}, \mathrm{NaCl},\left(\mathrm{NH}_{4}\right)_{2} \mathrm{SO}_{4}$, and $\mathrm{MgSO}_{4}$ was $17,33,89,99$, and 100 , respectively. Tris(hydroxymethyl)amino methane (Tris) buffer at $p \mathrm{H}$ 7.5 had no effect on the rate of lysis when tested at concentrations from 0.01 to $0.05 \mathrm{M}$. In Tris buffer, the optional $p \mathrm{H}$ for lysis is at 7.5, although the lysis does not change appreciably from $p \mathrm{H}$ 7.0 to 9.5. The results obtained in Tris and imidazole buffers are shown in Fig. 6 .

The finding that protoplasts of $B$. licheniformis are lysed by bacitracin suggested that this process might be the basis for the antibiotic activity of bacitracin. Since $B$. licheniformis is not a particularly sensitive organism, it was of interest to in-

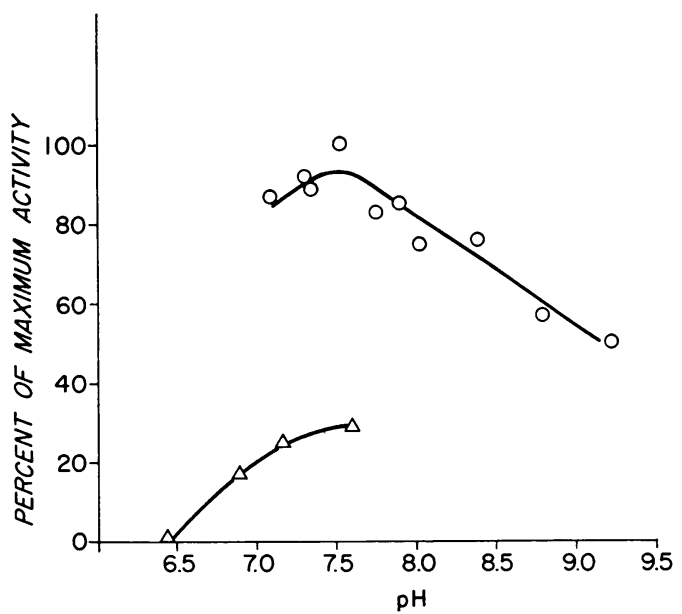

FIG. 6. Effect of $p H$ on the lysis of protoplasts by bacitracin. Protoplasts of Bacillus licheniformis were lysed by bacitracin (5.0 units per $\mathrm{ml}$ ) in the same manner as given in the legend for Fig. 4, except that the buffer was either $0.025 \mathrm{M}$ Tris (O) or $0.025 \mathrm{M}$ imidazole $(\triangle)$. The rate of lysis was estimated during the first minute after the addition of bacitracin. 


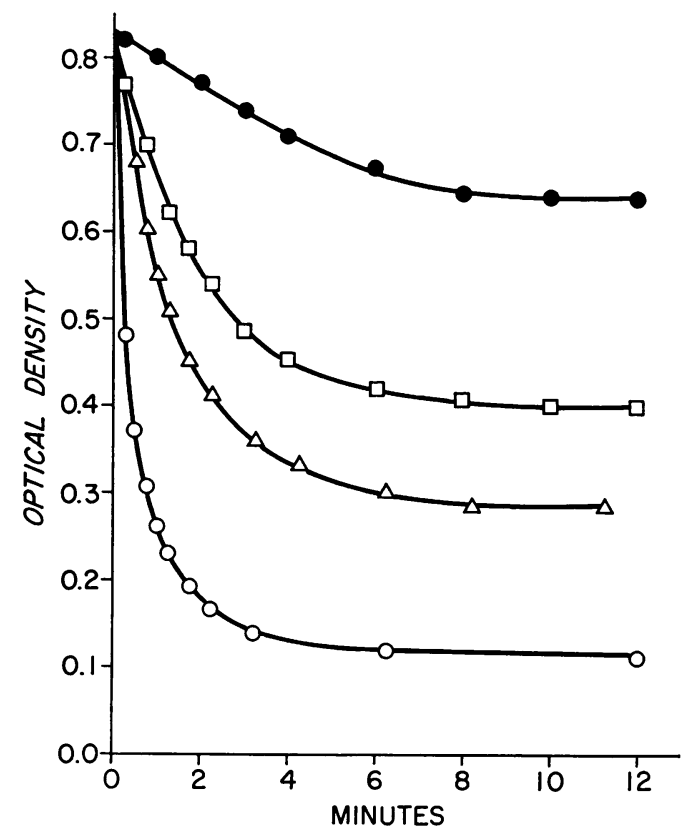

Fig. 7. Lysis of protoplasts of Micrococcus lysodeikticus by bacitracin. The incubation mixture, $4.0 \mathrm{ml}(\mathrm{pH} 8.5)$, contained: $1.0 \mathrm{M}$ sucrose, 0.025 M Tris buffer, $1.25 \times 10^{-4} \mathrm{M} \mathrm{CdCl}_{2}, 0.4 \mathrm{ml}$ of a protoplast suspension and bacitracin. The amount of antibiotic (units per milliliter) in each system was: $5.0(\mathrm{O})$, $2.5(\triangle), 1.25(\square)$, and $0.5(\bullet)$.

TABLE 2. Loss of bacitracin activities by alkaline treatment

\begin{tabular}{c|c|c}
\hline Time & $\begin{array}{c}\text { Antibacterial } \\
\text { activity* }\end{array}$ & Lytic activity \\
\hline $\min$ & $\%$ & $\%$ \\
10 & 50 & 40 \\
30 & 23 & 19 \\
60 & 13 & 10 \\
\hline
\end{tabular}

* Antibacterial activity was measured with Staphylococcus aureus, and the lytic activity was estimated by comparing the rate of lysis of Bacillus licheniformis protoplasts to the rates obtained with standard amounts of bacitracin.

vestigate the action of bacitracin on protoplasts of a more sensitive organism.

$M$. lysodeikticus is an organism which is both sensitive to bacitracin and is readily converted to protoplasts. When grown in Nutrient Broth, the concentration of bacitracin required to inhibit the growth of $M$. lysodeikticus is approximately 0.02 units per ml, 50 times smaller than that required to inhibit $B$. licheniformis.

The effect of adding bacitracin and cadmium ions to protoplasts of $M$. lysodeikticus is shown in
Fig. 7. The results demonstrate that in their susceptibility to lysis by bacitracin, protoplasts of $M$. lysodeikticus are approximately equivalent to those of $B$. licheniformis. Both the initial rates of lysis and the extent of lysis with varying amounts of bacitracin were comparable when the two different protoplasts were used.

It was of interest to determine whether the lytic and the antibacterial activities of bacitracin resided in the same chemical structure. Bacitracin, 280 units per ml, was inactivated by heating in a boiling-water bath at $p \mathrm{H} 11$. At various time intervals, samples were withdrawn, and neutralized with Tris buffer, and the two activities were determined. The results (Table 2) show that the rate of loss of the lytic activity closely parallels that of the microbiological activity, suggesting that the same chemical structure is probably involved in both activities.

\section{Discussion}

A consideration of antibiotics and their mode of action frequently leads to the question as to why any particular antibiotic is inactive against the microorganism which is responsible for its production. This inactivity would appear to be self-evident and is generally assumed to be the case. The results presented in this report demonstrate that the growth of $B$. licheniformis can be inhibited by bacitracin. The accumulation of large amounts of antibiotic in the later stages of a growing culture is possible, because the effect of the antibiotic is confined to the early growth phase. These results are very similar to those found for the neomycin-producing strain of Streptomyces fradiae, the growth of which can be inhibited by neomycin (Waksman, 1953).

The results presented in this report demonstrate that the antibiotic has a profound effect on the bacterial protoplast, and suggest the possibility that an alteration of the integrity of the protoplasmic membrane might be the basis of bacitracin antibiotic action. The high concentration of antibiotic used to induce protoplast lysis, approximately 200 times larger than that used to inhibit the growth of sensitive organisms, would not appear to be in accordance with the hypothesis that the site of bacitracin action is on the membrane. However, the number of protoplasts per milliliter in the lysis experiments was approximately 250 times that which would be derived from the number of cells per milliliter in the growth experiments. Thus, the ratios of cells to antibiotic in both the lysis and growth experiments are comparable. The possibility that the primary action of bacitracin might be on the membrane was also suggested by Hancock and Fitz-James (1964) in a report in which they dem- 
onstrated an effect of bacitracin on membranes of $B$. megaterium. If the site of action of bacitracin is on membranes, then the effect on cell-wall synthesis and protein synthesis might represent secondary effects of the antibiotic.

The susceptibility to lysis of protoplasts prepared from the sensitive $M$. lysodeikticus is similar to that of protoplasts obtained from the relatively resistant $B$. licheniformis. If it is assumed that the protoplast lysis indicates the site of bacitracin action, then the differences in sensitivity would reflect differences in the permeability of the cell walls to the antibiotic.

The antibiotic polymyxin, another of the peptide antibiotics, has its site of action on the lipoprotein membranes of susceptible cells (Newton, 1958). Since the peptide antibiotics are similar in both chemical structure and toxicity, it may be possible that the site of their antibiotic activity is the same, and that the disruption of lipoprotein membranes is also the basis for their toxicity.

\section{ACKNowledgment}

This investigation was supported by Public Health Service grant AI-05924 from the National Institute of Allergy and Infectious Diseases.

\section{Literature Cited}

Abraham, E. P. 1957. Biochemistry of some peptide and steroid hormones. John Wiley and Sons, Inc., New York.

Abraham, E. P., and G. G. F. Newton. 1958. Structure and function of some sulphur-containing peptides. Ciba Found. Symp. Amino Acids Peptides Antimetab. Activity, p. 205223.

Adler, R. H., ANd J. E. SNoke. 1962. Requirement of divalent metal ions for bacitracin activity. J. Bacteriol. 83:1315-1317.

Bernlohr, R. W., and G. D. Novelli. 1960. Some characteristics of bacitracin production of Bacillus licheniformis. Arch. Biochem. Biophys. $87: 232-238$.

Crawford, K., and E. P. Abraham. 1957. The synergistic action of cephalosporin $\mathrm{C}$ and ben- zyl-penicillin against a penicillinase-producing strain of Staphylococcus aureus. J. Gen. Microbiol. 16:604-613.

Creaser, E. H. 1955. The induced (adaptive) biosynthesis of $\beta$-galactosidase in Staphylococcus aureus. J. Gen. Microbiol. $12: 288-297$.

Darker, G. D., H. B. Brown, A. H. Free, B Biro, and J. T. Goorley. 1948. The assay of bacitracin. J. Amer. Pharm. Assoc. Sci. Ed. $37: 156-160$.

GaLe, E. F., AND J. P. Folkes. 1955. The assimilation of amino acids by bacteria. 21. The effect of nucleic acids on the development of certain enzymic activity in disrupted Staphylococcus cells. Biochem. J. 59:675-684.

Hancock, R., and P. C. Fitz-James. 1964. Some differences in the action of penicillin, bacitra cin, and vancomycin on Bacillus megaterium. J. Bacteriol. $87: 1044-1050$.

Hendis, D. 1949. The nutritional requirements of a bacitracin producing strain of Bacillus subtilis. Arch. Biochem. Biophys. 24:435-446.

Mandelstam, J., and H. J. Rogers. 1959. The incorporation of amino acids into the cell-wall mucopeptide of Staphylococci and the effect of antibiotics on the process. Biochem. J. 72:654662.

Newton, B. A. 1958. Surface active bactericides. Symp. Soc. Gen. Microbiol. 8:62-93.

$\mathrm{P}_{\text {ARK }}$, J. T. 1958. Inhibition of cell-wall synthesis in Staphylococcus aureus by chemicals which cause accumulation of wall precursors. Biochem. J. 70:2P.

PARK, J. T. 1960. Inhibition of synthesis of bacterial mucopeptides or protein by certain antibiotics and its possible significance for microbiology and medicine. Antimicrobial Agents Ann., p. 338-342.

Smith, J. L., ANd E. D. Weinberg. 1962. Mechanisms of antibacterial action of bacitracin. $\mathbf{J}$. Gen. Microbiol. 28:559-569.

SNOKE, J. E. 1961. Formation of bacitracin by protoplasts of Bacillus licheniformis. J. Bacteriol. 81:986-989.

WaKsman, S. A. 1953. Neomycin, p. 110. Rutgers University Press, New Brunswick, N.J.

WeinBerg, E. D. 1959. Enhancement of bacitracin by the metallic ions of group IIB. Antibiotics Ann. 1958-1959, p. 924-929. 